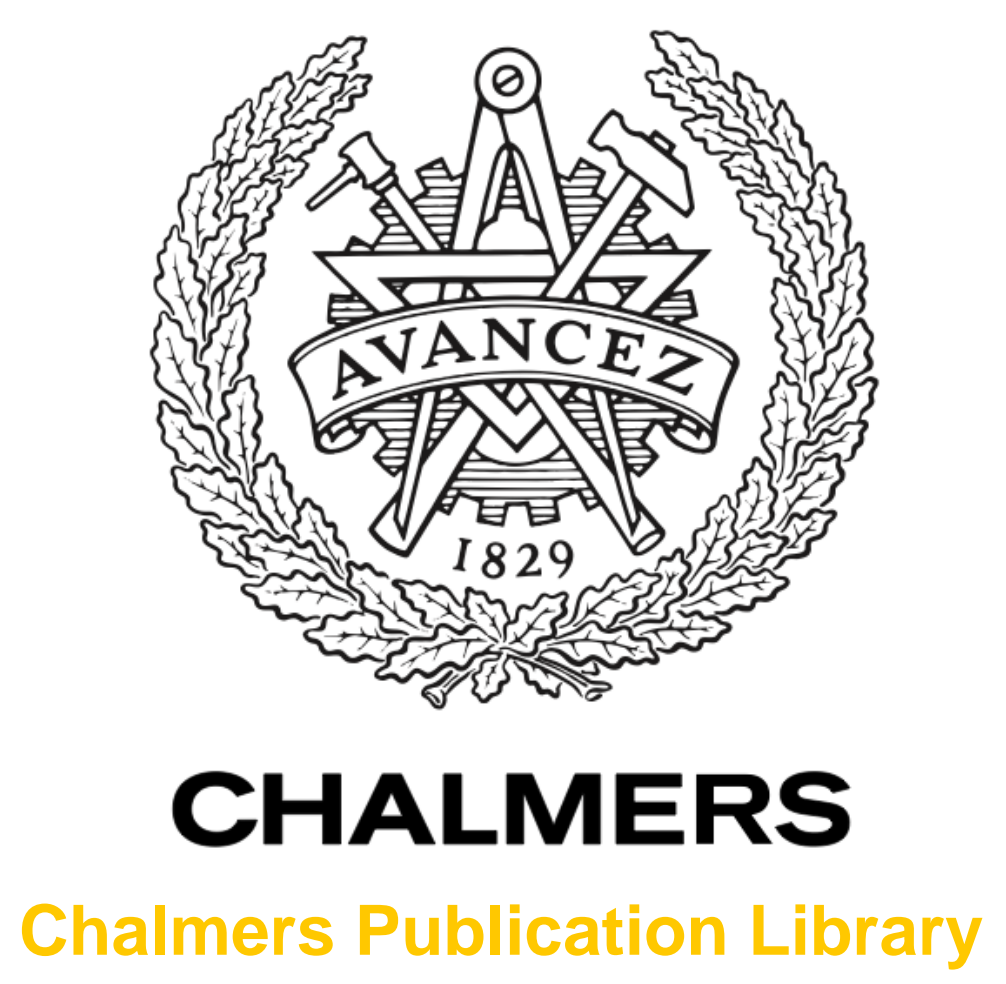

\title{
Measurement Uncertainty of Antenna Efficiency in a Reverberation Chamber
}

This document has been downloaded from Chalmers Publication Library (CPL). It is the author's version of a work that was accepted for publication in:

leee Transactions on Electromagnetic Compatibility (ISSN: 0018-9375)

Citation for the published paper:

Chen, X. (2013) "Measurement Uncertainty of Antenna Efficiency in a Reverberation

Chamber". Ieee Transactions on Electromagnetic Compatibility, vol. 55(6), pp. 1331-1334.

http://dx.doi.org/10.1109/temc.2013.2265042

Downloaded from: http://publications.lib.chalmers.se/publication/192784

Notice: Changes introduced as a result of publishing processes such as copy-editing and formatting may not be reflected in this document. For a definitive version of this work, please refer to the published source. Please note that access to the published version might require a subscription.

Chalmers Publication Library (CPL) offers the possibility of retrieving research publications produced at Chalmers University of Technology. It covers all types of publications: articles, dissertations, licentiate theses, masters theses, conference papers, reports etc. Since 2006 it is the official tool for Chalmers official publication statistics. To ensure that Chalmers research results are disseminated as widely as possible, an Open Access Policy has been adopted.

The CPL service is administrated and maintained by Chalmers Library. 


\title{
Letters
}

\section{Measurement Uncertainty of Antenna Efficiency in a Reverberation Chamber}

\author{
Xiaoming Chen
}

\begin{abstract}
Reverberation chambers have been used for measuring antenna efficiencies. In this letter, the measurement uncertainty of the efficiency measurement is analyzed and a simple uncertainty model is given. The model is verified by extensive measurements.
\end{abstract}

Index Terms-Antenna efficiency, independent sample number, measurement uncertainty, reverberation chamber (RC).

\section{INTRODUCTION}

$\mathbf{T}$ $\mathrm{HE}$ reverberation chamber $(\mathrm{RC})$ has traditionally been used for electromagnetic compatibility tests [1]. Over the past decade, it has found applications in various over-the-air (OTA) tests such as measuring antenna efficiencies [2], diversity gains and ergodic capacities of multiantenna systems [3], and bit error rates of telecommunication systems [4], [5]. Almost all of these applications necessitate a reference measurement (to determine the average power transfer function $P_{\text {ref }}$ [6]) for calibrating the chamber. Therefore, the measurement accuracy of $P_{\text {ref }}$ affects the measurement accuracies of all the OTA tests. The measurement uncertainty of $P_{\text {ref }}$ was studied in [6] and [7]. However, the actual uncertainty effects of $P_{\text {ref }}$ on OTA tests have not been studied quantitatively yet.

In this letter, we will study the measurement uncertainty of antenna efficiency in an RC. In the literature, there are quite a few different methods for measuring antenna efficiency in an RC, e.g., [8]-[11]. A time-reversal method was presented in [8] for measuring the antenna efficiency in the time domain. However, the time-reversal method is limited to ultrawideband antennas. To eliminate the use of a reference antenna [2], [6], different approaches were introduced in [9]-[11]. In [9], antenna efficiencies were measured by reflection measurements, which sometimes suffer poor accuracy in the return loss. By using two identical antennas under test (AUT), the antenna efficiency can be measured by estimating the quality factor of the chamber [10]. Several methods for measuring antenna efficiencies by estimating the chamber decay time were presented in [11]. Except for the empirical uncertainty study in [11], efficiency measurement uncertainties were generally overlooked in these studies. In this letter, measurement uncertainties of antenna efficiencies will be studied and modeled for the standard antenna

Manuscript received May 7, 2013; accepted May 10, 2013. Date of publication June 11, 2013; date of current version December 10, 2013.

The author is with the Department of Signals and Systems, Chalmers University of Technology, 41258 Gothenburg, Sweden (e-mail: xiaoming.chen@ chalmers.se).

Color versions of one or more of the figures in this paper are available online at http://ieeexplore.ieee.org.

Digital Object Identifier 10.1109/TEMC.2013.2265042 efficiency method, e.g., [2], [12]. (Note that the uncertainty analysis in [12] is actually for $P_{\text {ref }}$ instead of antenna efficiency.) The uncertainty model is verified by extensive measurements in an RC.

\section{MEASUREMENT UNCERTAINTY OF ANTENNA EFFICIENCY}

The standard method for measuring the antenna efficiency is to first measure the average power transfer function $P_{\text {ref }}$ of a reference antenna (with known antenna efficiency $e_{\text {ref }}$ ) and then measure that of the AUT, $P_{\mathrm{AUT}}$. During both measurements, both the reference antenna and the AUT must be placed in the chamber in order to keep the same RC loading [7]. The total radiation efficiency of the AUT can be estimated as

$$
e_{\mathrm{AUT}}=\frac{P_{\mathrm{AUT}}}{P_{\mathrm{ref}} / e_{\mathrm{ref}}}=\frac{X}{Y}
$$

where $Y=P_{\text {ref }} / e_{\text {ref }}$ (normalized average power transfer function of the reference antenna) and $X=P_{\mathrm{AUT}}$ (average power transfer function of the AUT).

Denote $\mu_{x}=E[X], \mu_{y}=E[Y]$, where $E$ denotes expectation, and $f(x, y)=e_{\mathrm{AUT}}(X=x, Y=y)$. Taking the first-order Taylor expansion of (1), one obtains

$f(x, y) \approx f\left(\mu_{x}, \mu_{y}\right)+\left.\frac{\partial f}{\partial x}\right|_{\substack{x=\mu_{x} \\ y=\mu_{y}}}\left(x-\mu_{x}\right)+\left.\frac{\partial f}{\partial y}\right|_{\substack{x=\mu_{x} \\ y=\mu_{y}}}\left(x-\mu_{y}\right)$.

The variance of $e_{\mathrm{AUT}}$ is

$$
\begin{aligned}
\operatorname{var}\left[e_{\mathrm{AUT}}\right] \approx & E\left[\left(f(x, y)-f\left(\mu_{x}, \mu_{y}\right)\right)^{2}\right] \\
\approx & \left(\left.\frac{\partial f}{\partial x}\right|_{\substack{x=\mu_{x} \\
y=\mu_{y}}}\right)^{2} E\left[\left(x-\mu_{x}\right)^{2}\right] \\
& +\left(\left.\frac{\partial f}{\partial y}\right|_{\substack{x=\mu_{x} \\
y=\mu_{y}}}\right)^{2} E\left[\left(y-\mu_{y}\right)^{2}\right] \\
& +\left.\left.2 \frac{\partial f}{\partial x}\right|_{\substack{x=\mu_{x} \\
y=\mu_{y}}} \frac{\partial f}{\partial y}\right|_{\substack{x=\mu_{x} \\
y=\mu_{y}}} E\left[\left(x-\mu_{x}\right)\left(y-\mu_{y}\right)\right] \\
= & \left(\frac{1}{\mu_{y}}\right)^{2} \operatorname{var}[X]+\left(\frac{-\mu_{x}}{\mu_{y}^{2}}\right)^{2} \operatorname{var}[Y] \\
& +\left.\left.2 \frac{\partial f}{\partial x}\right|_{\substack{x=\mu_{x} \\
y=\mu_{y}}} \frac{\partial f}{\partial y}\right|_{\substack{x=\mu_{x} \\
y=\mu_{y}}} \operatorname{cov}[X, Y] \\
= & \left(\frac{\mu_{x}}{\mu_{y}}\right)^{2}\left(\frac{\operatorname{var}[X]}{\mu_{x}^{2}}+\frac{\operatorname{var}[Y]}{\mu_{y}^{2}}\right)^{2}
\end{aligned}
$$


where var represents the variance and cov denotes the crosscovariance. The last equality holds under the assumption that $X$ and $Y$ are independent. Note that this is not a strong assumption in that the average power transfer functions of the reference antenna and the AUT are obtained via two independent measurements.

The average power transfer functions $Y$ is the average of $N_{\text {ind }}$ independent and identically distributed (i.i.d.) power transfer functions of the reference antenna (normalized by $e_{\text {ref }}$ ). In a well-stirred RC, these i.i.d. power transfer functions are exponentially distributed [1] with a mean $P_{0}$. As a result

$$
\begin{aligned}
\mu_{y} & =P_{0}, \\
\operatorname{var}[Y] & =P_{0} / N_{\text {ind }} .
\end{aligned}
$$

Denote $E\left[e_{\mathrm{AUT}}\right]=e_{0}$. Note that i.i.d. exponentially distributed power transfer function seen by the AUT is attenuated by $e_{0}$. Thus, the mean of the power transfer faction is $e_{0} P_{0}$. Similarly, one obtains

$$
\begin{aligned}
\mu_{x} & =e_{0} P_{0}, \\
\operatorname{var}[X] & =\frac{e_{0} P_{0}}{N_{\text {ind }}} .
\end{aligned}
$$

Substituting (4) and (5) into (3), one obtains

$$
\operatorname{var}\left[e_{\mathrm{AUT}}\right] \approx \frac{2 e_{0}^{2}}{N_{\mathrm{ind}}}
$$

The standard deviation (STD) of $e_{\mathrm{AUT}}$ is

$$
\operatorname{std}\left[e_{\mathrm{AUT}}\right] \approx e_{0} \sqrt{\frac{2}{N_{\text {ind }}}}
$$

where std denoting the STD. The independent sample number $N_{\text {ind }}$ can be estimated (based on one reference measurement) using, e.g., the degree-of-freedom (DoF) method [7].

As can be seen in the next section, the estimated $N_{\text {ind }}$ using the DoF method takes the RC loading into account. It is known that RC loading (without blocking the line-of-sight path between transmitting and receiving antennas) increases the ratio of the unstirred power to the stirred power. Thus, the uncertainty due to the unstirred component is included in the uncertainty model (7). Note that this letter does not consider the systematic uncertainty, which should be investigated in the future work.

\section{MeAsurements}

In order to verify the efficiency uncertainty model (7), extensive measurements were performed from 700 to $3000 \mathrm{MHz}$ (covering the most interesting telecommunication bands) in the Bluetest HP RC [6]. The RC used has a size of $1.80 \mathrm{~m} \times$ $1.75 \mathrm{~m} \times 1.25 \mathrm{~m}$ (a drawing of which is shown in Fig. 1). It has two mode-stirring plates, a turn-table platform (on which a wideband discone antenna, used as the reference antenna, is mounted), and three half-bow-tie antennas mounted on three orthogonal walls (referred to as wall antennas hereafter). During the measurement, the turn-table platform was moved stepwise to 20 platform-stirring positions (evenly distributed over one complete platform rotation); at each platform-stirring position, the two plates were moved simultaneously and stepwise to 50

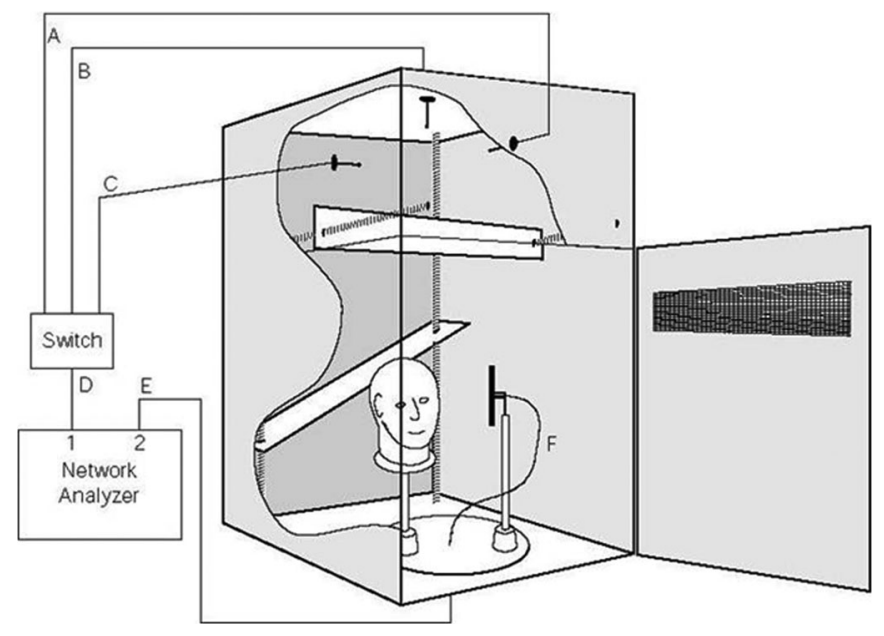

Fig. 1. Drawing of the RC with two mechanical plate stirrers, a platform, three wall antennas, and a head phantom.

positions (equally spanned on the total distances that they can travel along two walls inside the RC). At each stirrer position and for each wall antenna, a full frequency sweep was performed by a vector network analyzer with a frequency step of $1 \mathrm{MHz}$, during which the scattering parameters (S-parameters) are sampled (as a function of frequency and stirring position). Hence, for each measurement, we have three wall antennas, 50 plate-stirring positions, and 20 platform-stirring positions.

To facilitate the estimation of the antenna efficiency uncertainty and without loss of generality, the same measurement sequence is repeated 12 times, each time with a different height/orientation of the reference antenna on the platform, i.e., the reference antenna was placed at four different heights and at each height it is placed with one vertical and two horizontal orientations (in radial and tangential directions of the platform), respectively. The heights and orientations are chosen to ensure independent measurements. In postprocessing, arbitrary pairs of antenna heights/orientations are chosen as the AUTs and the reference antennas, respectively, for estimating $e_{\mathrm{AUT}}$, and we introduce 0-, 1-, 2-, and 3-dB attenuator to the AUT (whose negative value in decibels is the expectation of $e_{\mathrm{AUT}}$ ).

Note that it is nontrivial to find the maximum number of independent $e_{\mathrm{AUT}}$ samples from the 12 measurements due to the complicated distribution of $e_{\mathrm{AUT}}$, i.e., the ratio of two nonzero Gaussian variables [13]. To be safe, we choose six pairs of distinct measurements to obtain six independent $e_{\mathrm{AUT}}$, which are used for estimating the measurement uncertainty of $e_{\mathrm{AUT}}$.

In order to see the RC loading effect on the measurement uncertainty of $e_{\mathrm{AUT}}$, the measurement procedure was repeated for two loading configurations: load0 (unloaded RC) and loadl (a head phantom that is equivalent to a human head in terms of microwave absorption). Hereafter, measured data from these different loading configurations are simply referred to as load0 and loadl data, whose quality factors are around 1000 and 550, respectively. Hence, the STD of $e_{\mathrm{AUT}}$ is estimated from these six $e_{\mathrm{AUT}}$ samples (for each $e_{0}$ case and each loading configuration) at each frequency point. Since $N_{\text {ind }}$ can be estimated based on one reference measurement using the DoF method [7], the 


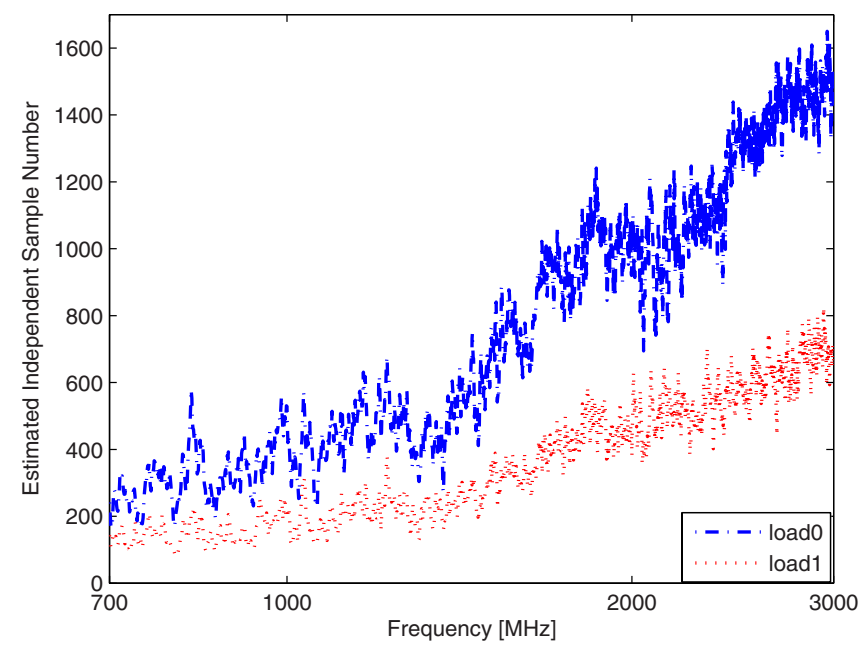

Fig. 2. Estimated independent sample number $N$ using the DoF method.
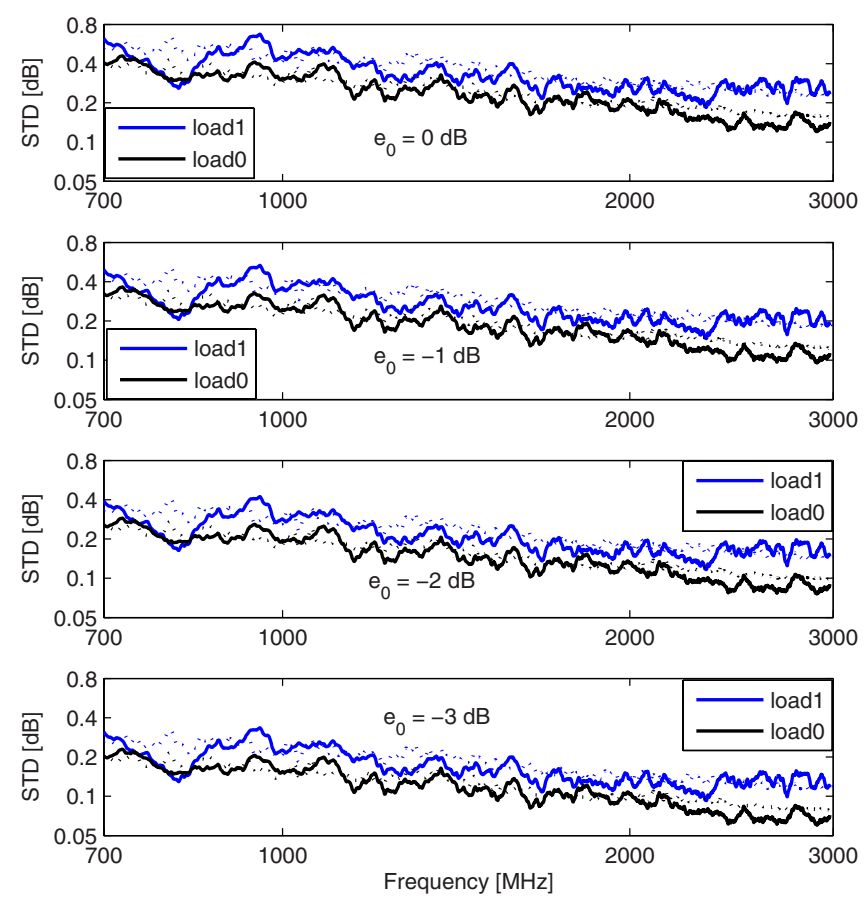

Fig. 3. Estimated antenna efficiency uncertainties based on nine independent measurements (solid) and the uncertainty model (7) (dotted). The four graphs from top to bottom correspond to $e_{0}=0,-1,-2$, and $-3 \mathrm{~dB}$, respectively, where $e_{0}=E\left[e_{\mathrm{AUT}}\right]$.

$e_{\mathrm{AUT}} \mathrm{STD}$ can also be estimated using the uncertainty model (6). Fig. 2 shows the estimated $N_{\text {ind }}$ as a function of frequency for both load0 and loadl.

Fig. 3 shows the estimated STDs based on the 12 independent measurements and the uncertainty model (7) from a single measurement (with estimated $N_{\text {ind }}$ using the DoF method [7]), respectively. Note that the DoF method inherently takes the RC loading (and therefore K-factor [6]) effect into account [7]. Therefore, there is no need in decomposing the STD into stirred and unstirred components as in [6]. Also note that, for clear exhibitions, the estimated STD is plotted using the following
dB-transformation [6]

$$
\sigma_{\mathrm{dB}}=5 \log _{10} \frac{(1+\sigma)}{(1-\sigma)}
$$

and that a 50-MHz frequency smoothing is applied to the STD curves before plotting. As can be seen, there is good agreement between the uncertainty model (based on one measurement) and the direct STD estimate (based on the 12 independent measurements). It can also be seen that $e_{\mathrm{AUT}}$ measurement uncertainty decreases with decreasing total radiation efficiency of the AUT. The later observation can be readily explained from $e_{\mathrm{AUT}}$ uncertainty model (7).

Note that the DoF method for estimating $N_{\text {ind }}$ proposed in [7] is suitable to RCs with different (separable) stirring mechanisms. In case the stirring sequences generated by different stirring mechanisms are not separable, or there is only one stirring mechanism, one can either use the frequency-domain samples for estimating $N_{\text {ind }}$ (at the cost of sever degradation of the frequency resolution) [14] or simply use suitable $N_{\text {ind }}$ estimators, e.g., [15], [16].

\section{CONCLUSION}

In this letter, the measurement uncertainty of antenna efficiency estimated using the standard efficiency measurement approach [2] is analyzed. A simple efficiency uncertainty model (approximation) is given. And the model is verified based on extensive RC measurements. The good agreement indicates that the model can be used for predicting the measurement uncertainty of the antenna efficiency based on a single reference measurement.

\section{REFERENCES}

[1] J. G. Kostas and B. Boverie, "Statistical model for a mode-stirred chamber," IEEE Trans. Electromagn. Compat., vol. 33, no. 4, pp. 366-370, Nov. 1991.

[2] EMC, Part 4: Testing and Measurement Techniques; Section 21: Reverberation Chamber Test Methods, Int. Electrotech Comm., Geneva, Switzerland, Committee Draft, IEC 61000-4-21, 2001.

[3] X. Chen, P.-S. Kildal, J. Carlsson, and J. Yang, "MRC diversity and MIMO capacity evaluations of multi-port antennas using reverberation chamber and anechoic chamber," IEEE Trans. Antennas Propag., vol. 61, no. 2, pp. 917-926, Feb. 2013.

[4] M. Á. García-Fernández, J. D. Sánchez-Heredia, A. M. MartínezGonzález, D A. Sánchez-Hernández, and J. F. Valenzuela-Valdés, "Advances in mode-stirred reverberation chambers for wireless communication performance evaluation," IEEE Commun. Mag., vol. 49, no. 7, pp. 140-147, Jul. 2011.

[5] G. Ferrara, M. Migliaccio, and A. Sorrentino, "Characterization of GSM non-line-of-sight propagation channels generated in a reverberating chamber by using bit error rates," IEEE Trans. Electromagn. Compat., vol. 49, no. 3, pp. 467-473, Aug. 2007.

[6] P.-S. Kildal, X. Chen, C. Orlenius, M. Franzén, and C. Lötbäck Patané "Characterization of reverberation chambers for OTA measurements of wireless devices: Physical formulations of channel matrix and new uncertainty formula," IEEE Trans. Antennas Propag., vol. 60, no. 8, pp. 3875 3891, Aug. 2012.

[7] X. Chen, "Experimental investigation of the number of independent samples and the measurement uncertainty in a reverberation chamber," IEEE Trans. Electromagn. Compat., , to be published.

[8] G. L. Fur, P. Besnier, and A. Sharaiha, "Time reversal efficiency measurement in reverberation chamber," IEEE Trans. Antennas Propag., vol. 60, no. 6, pp. 2921-2928, Jun. 2012. 
[9] P. Hallbjörner, "Reflective antenna efficiency measurements in reverberation chambers," Microw. Opt. Technol. Lett., vol. 30, no. 5, pp. 332-335, 2001.

[10] H. G. Krauthauser and M. Herbrig, "Yet another antenna efficiency measurement method in reverberation chambers," in Proc. IEEE Int. Symp. Electromagn. Compat., Jul. 25-30, 2010, pp. 536-540.

[11] C. L. Holloway, H. Shah, R. J. Pirkl, W. Young, J. Ladbury, and D. A. Hill, "Reverberation chamber techniques for determining the radiation and total efficiency of antennas," IEEE Trans. Antennas Propag.., vol. 60, no. 4, pp. 1758-1770, Apr. 2012.

[12] S. J Boyes, Y. Huang, N. Khiabani, P. J. Soh, and G. A. E. Vandenbosch, "Repeatability and uncertainty evaluations of on-body textile antenna efficiency measurements in a reverberation chamber," in Loughborough Antennas Propag. Conf., Loughborough, U.K., Nov. 12-13, 2012, pp. 1-5.
[13] D. V. Hinkely, "On the ratio of two correlated normal variables," Biometrika, vol. 56, no. 3, pp. 635-639, Dec. 1969.

[14] R. J. Pirkl, K. A. Remley, and C. S. L. Patané, "Reverberation chamber measurement correlation," IEEE Trans. Electromagn. Compat.,, vol. 54, no. 3, pp. 533-544, Jun. 2012.

[15] C. Lemoine, P. Besnier, and M. Drissi, "Estimating the effective sample size to select independent measurements in a reverberation chamber,' IEEE Trans. Electromagn. Compat., vol. 50, no. 2, pp. 227-236, May 2008.

[16] G. Gradoni, V. M. Primiani, and F. Moglie, "Reverberation chamber as a multivariate process: FDTD evaluation of correlation matrix and independent positions," Progr. Electromagn. Res., vol. 133, pp. 217-234, 2013. 